\title{
Searches for narrow resonances decaying to pairs of boosted $\mathrm{HH}$ bosons
}

\author{
Angelo Santos*† \\ For the CMS Collaboration \\ Universidade Federal do ABC (UFABC) \\ E-mail: angelo.santos@cern.ch
}

\begin{abstract}
We present a search for narrow resonances production in gluon-gluon fusion followed by a decay into two Higgs bosons at $8 \mathrm{TeV}$. Decays of Higgs bosons into bottom quark pairs are considered for resonance masses above $1 \mathrm{TeV}$, where each Higgs boson is produced with large momentum, and the hadronization products of the pair of bottom quarks can usually be reconstructed as single large jets. The background from multijet and $t \bar{t}$ events is significantly reduced by applying requirements related to the flavor of the jet, its mass, and its substructure. The signal is identified as a peak in the dijet invariant mass spectrum of the remaining background events.
\end{abstract}

38th International Conference on High Energy Physics

3-10 August 2016

Chicago, USA

\footnotetext{
* Speaker.

$\dagger$ This material is based upon work supported by the São Paulo Research Foundation (FAPESP) under Grant No. 2013/01907-0.
} 


\section{Introduction}

Many BSM models predict the existence of massive particle $X$ that can decay in pairs of Higgs bosons. In the context of warped extra dimension models [1,2,3], this particle could be a scalar radion, a resonance of spin- 0 associated with fluctuations in the length of the extra dimensions. The production cross section of the theory is proportional to the inverse of $\Lambda_{R}$, the parameter scale. Considering the radion production from gluon-gluon fusion, with $B(X \rightarrow H H) \approx 25 \%$ at $1 \mathrm{TeV}$, we perform a search for a radion decaying in a pair of Higgs bosons. A final state of $H H \rightarrow b \bar{b} b \bar{b}$ is taken into account, which benefits from a branching fraction of $B(H H \rightarrow b \bar{b} b \bar{b}) \approx 57 \%$.

In this study, we analyze a total integrated luminosity of $19.7 \mathrm{fb}^{-1}$ of data collected by the CMS experiment [4] provided by proton-proton collisions in a center-of-mass of $8 \mathrm{TeV}$ at the LHC. Since we are interested in a boosted regime, we consider radion masses of $m_{\mathrm{X}}>1 \mathrm{TeV}$, so that the jets produced with the hadronization of each bottom quark are characterized by large momentum $p_{\mathrm{T}}$ and appear as a single fat jet. The multijet background is dominated by QCD and $t \bar{t}$ samples, turning out to be a challenge to identify the signal of radion. Figure 1 (on the left) shows the masses of the fat jets from the decays of each Higgs boson. The signal appears in the falling tail of observed and background events.
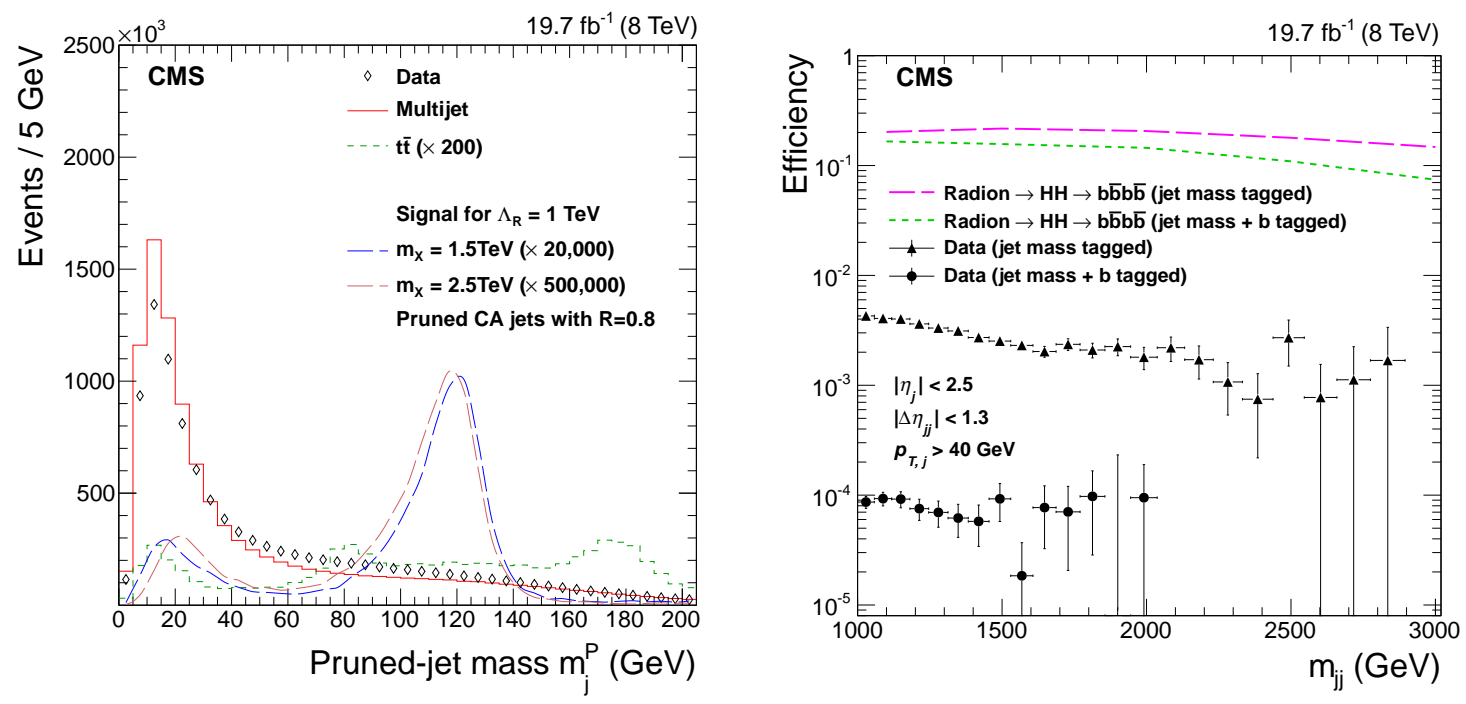

Figure 1: Fat jet mass distribution (left) from each Higgs boson for observed (dots) and background events (histograms). Signal of radion for different mass points are shown as smooth histograms. Selection efficiency (right) for signal and observed events considering the number of b-tags and fat jets with mass around the Higgs boson mass

Data analysis techniques are used to resolve the hadronization products from the bottom quarks in order to optimize the signal against the background. The jet substructure and "b-tagging" [5] techniques allow to identify and distinguish decay products from the bottom quarks. The "Higgstagging" [6] enhances the probability to get events whose jets have origin in the Higgs bosons. In addition, selections related to the fiducial acceptance are applied to the events: at least two jets with $p_{\mathrm{T}}>40 \mathrm{GeV}$; each jet with pseudo-rapidity $|\eta|<2.5$; angular separation between the fat jets along the pseudo-rapidity $\left|\Delta \eta_{\mathrm{jj}}\right|<1.3$; and invariant mass of the two fat jets (dijet) $m_{\mathrm{jj}}>1 \mathrm{TeV}$. After 
applying these selections, figure 1 (on the right) shows the efficiency of signal and observed events considering constraints in the Higgs boson mass and in the number of b-tags. The probability to identify events from two Higgs bosons appear to have no dependence with the dijet mass.

\section{Results}

Depending on the probability to resolve between one or two jets, the fat jets are classified as "high-purity" (HP) or "low-purity" (LP) respectively. Combining the possibilities leads to three different type of events: HP-HP, HP-LP, LP-HP. A data-derived method is used to estimate the multijet background, for each category, as a function of the dijet mass according to the fit function

$$
\frac{d N_{\mathrm{Background}}}{d m_{\mathrm{jj}}}=N_{\mathrm{B}} a e^{-a\left(m_{\mathrm{jj}}-1000 \mathrm{GeV}\right)} .
$$

The background shape is given by the parameter $a$ applying the fit function on the dijet mass $m_{\mathrm{jj}}$ distribution from observed events with fat jets of invariant mass $60<m_{\mathrm{j}}<100 \mathrm{GeV}$ (control region), as shown in Fig. 2 (left). Parameter $N_{\mathrm{B}}$ is related to the background normalization, which is obtained applying the same fit function on the dijet mass from events with $110<m_{\mathrm{j}}<135 \mathrm{GeV}$ (Higgs boson mass region), but fixing the slope $a$ from the control region, as shown in Fig. 2.
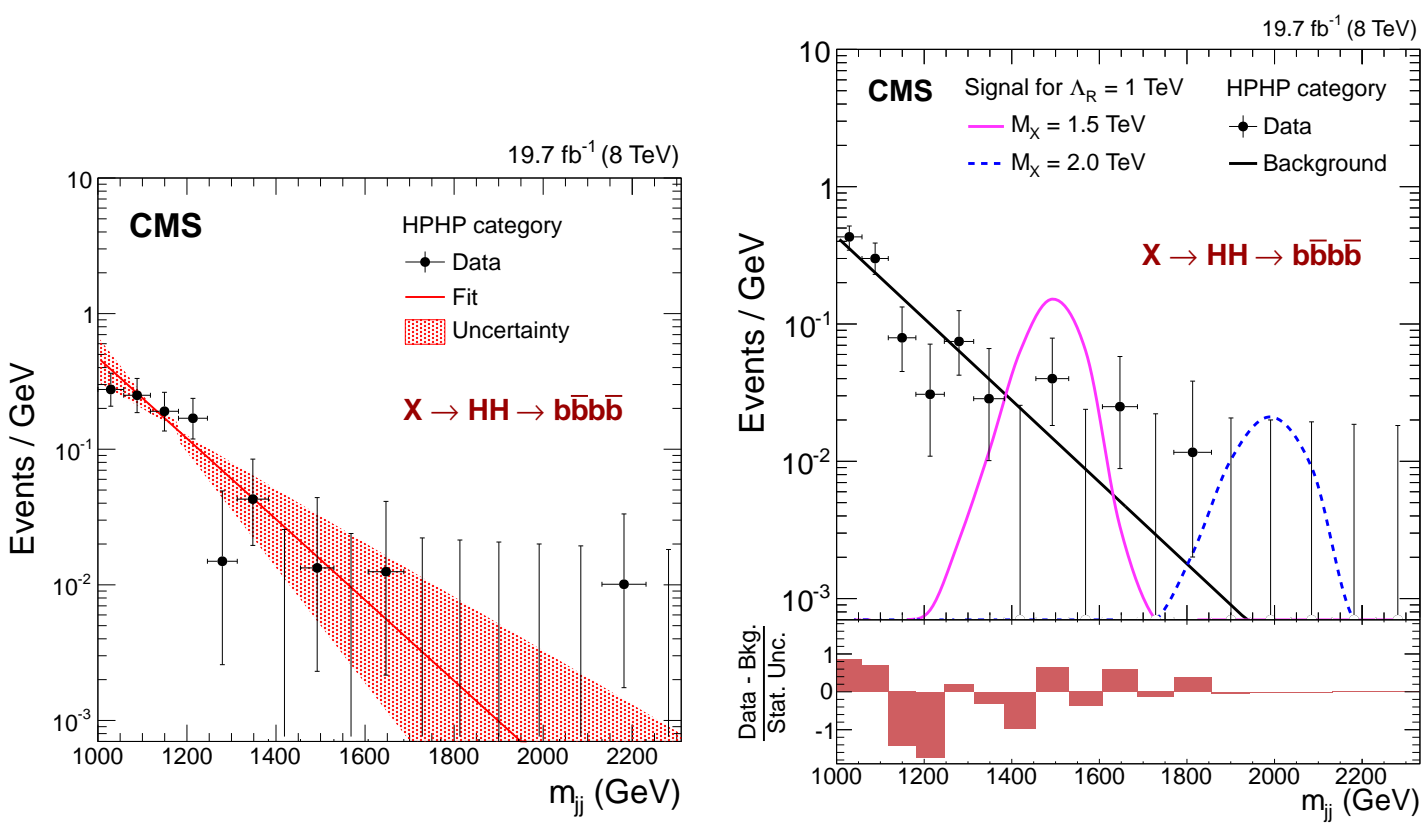

Figure 2: Dijet mass distributions from observed events (dots). Figure on the left shows distribution from control region, and the red band corresponds to the uncertainty from the slope of the fit function (red line). Figure on the right shows the dijet mass in the case of signal region (Higgs boson mass), and the normalization of the fit function (black line) takes the shape from the control region into account. These distributions are based on the HP-HP category. Signal distributions are shown for $M_{\mathrm{X}}=1$ (pink straight line) and $2 \mathrm{TeV}$ (blue dashed line). 


\section{Conclusion}

Since no excess of events are observed with respect to the expectations of the standard model, 95\% confidence level upper limits are set on the production cross section for $\Lambda_{R}=1 \mathrm{TeV}$ in the range of $1.15<m_{\mathrm{X}}<1.55 \mathrm{TeV}$, as shown in fig. 3, which has a conbination of all HP-HP, HPLP and LP-HP categories. In this figure, the values obtained with this study are compared with previous CMS results in the same channel [7]. The search of this report is published in the EPJC [8].

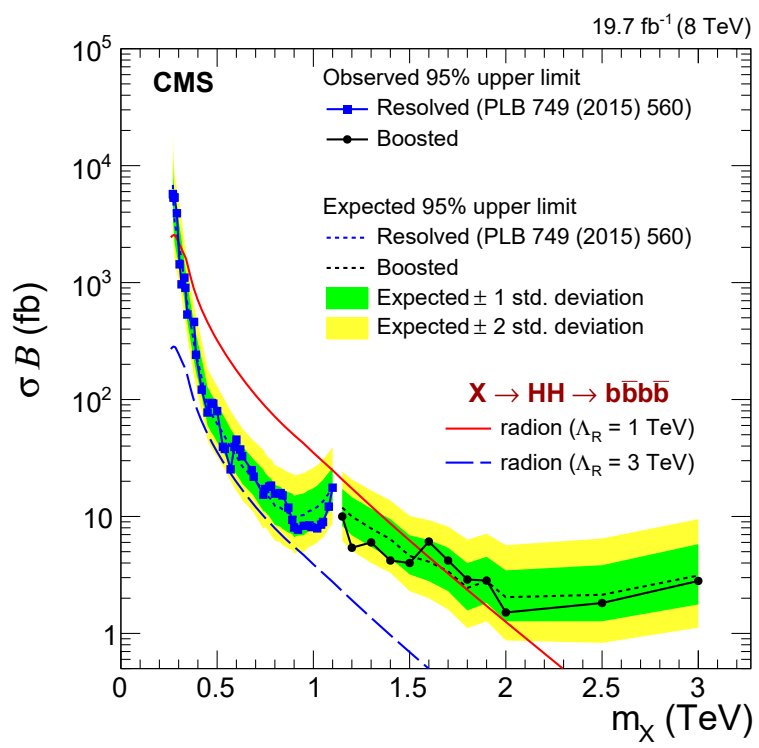

Figure 3: Observed and expected limits on the production cross section times the branching fraction as a function of $m_{\mathrm{X}}$. The theoretical curves are also shown for the warped extra dimension model in case of $\Lambda_{\mathrm{R}}=1$ (red straight line) and $\Lambda_{\mathrm{R}}=3 \mathrm{TeV}$ (blue dashed line). The limits from this study (black dots) are compared with the limits from previous CMS results (blue dots) in the same channel.

\section{References}

[1] W. Goldberger and M. Wise, Modulus stabilization with bulk fields, Phys. Rev. Lett. 84 (1992) 4922.

[2] O. DeWolfe, D. Freedman, S. Gubser and A. Karch, Modeling the fifth-dimension with scalars and gravity, Phys. Rev. D 62 (2000) 046008.

[3] C. Csaki, M. Graesser, L. Randall and J. Terning, Cosmology of brane models with radion stabilization, Phys. Rev. D 62 (2000) 045015.

[4] CMS Collaboration, The CMS experiment at the CERN LHC, JINST 3 (2008) S08004

[5] CMS Collaboration, Identification of b-quark jets with the CMS experiment, JINST 8 (2013) P04013.

[6] CMS Collaboration, Search for massive resonances decaying into pairs of boosted bosons in semi-leptonic final states at $\sqrt{s}=8 \mathrm{TeV}$, JHEP 08 (2014) 174.

[7] CMS Collaboration, Search for resonant pair production of Higgs bosons decaying to two bottom quark-antiquark pairs in proton-proton collisions at 8 TeV, Phys. Lett. B 749 (2015) 560.

[8] CMS Collaboration, Search for heavy resonances decaying to two Higgs bosons in final states containing four b quarks, Eur. Phys. J. C 76 (2016) 7, 371. 